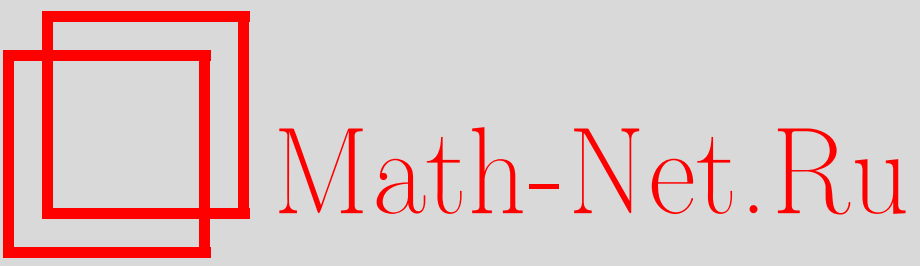

D. Dehay, Local time and convergence of empirical estimators, Теория вероятн. и ее примен., 2012, том 57, выпуск 2, 337-352

DOI: https://doi.org/10.4213/tvp4450

Использование Общероссийского математического портала Math-Net.Ru подразумевает, что вы прочитали и согласны с пользовательским соглашением

http://www.mathnet.ru/rus/agreement

Параметры загрузки:

IP: 3.89 .197 .203

26 апреля 2023 г., 14:31:14 


\title{
LOCAL TIME AND CONVERGENCE OF EMPIRICAL ESTIMATORS
}

\begin{abstract}
Пусть $\Phi_{T}=\left\{\Phi_{T}(x), x \in \mathbf{R}\right\}, T>0$, - семейство измеримых действительнозначных процессов на $\mathbf{R}$ с траекториями в $\mathscr{B}_{\mathrm{b}}$ (где $\mathscr{B}_{\mathrm{b}}$ - класс действительнозначных борелевских функций), которые при $T \rightarrow \infty$ сходятся в $\mathscr{B}_{\mathrm{b}}$ по распределению к некоторому процессу $\Phi$. Цель настоящей заметки - доказать сходимость взвешенных средних величин $\Phi_{T}\left(X_{t}\right)$ на $[0, T]$ при $T \rightarrow \infty$, где $\left(X_{t}\right)_{t \geqslant 0}-$ действительнозначный процесс, имеющий локальное время. Наш метод основан на стохастической версии формулы для времени пребывания, использующей разложение Карунена-Лоэва. Мы описываем одно применение этого результата для оценки инвариантного маргинального распределения эргодического диффузионного процесса.
\end{abstract}

Ключевые слова и фразы: локальное время, формула для времени пребывания, разложение Карунена-Лоэва, сходимость по распределению.

1. Introduction. Let $\Phi_{T}:=\left\{\Phi_{T}(x), x \in \mathbf{R}\right\}, T>0$, be a family of measurable real-valued processes on a probability space $(\Omega, \mathscr{F}, \mathbf{P})$ with paths in $\mathscr{B}_{\mathrm{b}}$, the class of real-valued Borel bounded functions (or in some space of real-valued functions), and which converges in distribution in $\mathscr{B}_{\mathrm{b}}$ to a process $\Phi$ as $T$ goes to $\infty$. The main purpose of this work is to present a method to obtain the convergence of the average of $\Phi_{T}\left(X_{t}\right)$ on $[0, T]$ as $T$ goes to $\infty$, where $X:=\left\{X_{t}, t \geqslant 0\right\}$ is a real-valued process (with a local time). More precisely, in this paper we introduce a smoothing function $g: \mathbf{R} \rightarrow \mathbf{R}$ and study the behavior of the weighted average

$$
\frac{1}{T} \int_{0}^{T} \Phi_{T}\left(X_{t}\right) g\left(X_{t}\right) \mathrm{d} t
$$

It is worthy to notice that we assume no dependency condition between the processes $X$ and $\Phi_{T}$.

*Institut de Recherche Mathématique de Rennes, CNRS, UMR 6625, Université Rennes 2, Rennes, France; e-mail: dominique.dehay@univ-rennes2.fr 
For this study we first generalize the occupation time formula to our context. This is done using the uniform in probability Karhunen-Loève expansion and the classical local time formula. Thus we transform our problem to the asymptotic behavior of the integral

$$
\int_{\mathbf{R}} \Phi_{T}(x) g(x) \frac{l_{T}(x)}{T} \mathrm{~d} x
$$

as $T \rightarrow \infty$, where $l_{T}$ is the local time of the process $\left\{X_{t}, t \geqslant 0\right\}$ on the interval $[0, T]$. Then we establish the convergence in distribution of the average (1) as a consequence of the generalized local time formula and the convergence properties in functional separable metric spaces [1].

The convergence of the empirical distribution for an ergodic diffusion process has been studied by I. Negri [11] (see also [9, Section 4.1]). Here we complete this work by analyzing the asymptotic behavior of integral functionals of the empirical distribution of an ergodic diffusion process.

Synopsis of the paper. In Section 2 we recall the notion of local time and the occupation time formula, as well the well-known Karhunen-Loève series expansion. With these tools we establish, in Section 3, a stochastic version of the occupation time formula, that is, we show the $\mathbf{P}$-almost everywhere (a.e.) equality between expressions (1) and (2). In Section 4 we deduce the convergence in distribution of the weighted average (1). Section 5 deals with the application to the problem of estimation of the distribution function of an ergodic diffusion process.

2. Background. First recall some usefull features concerning the notions of occupation time, local time and the Karhunen-Loève series expansion.

2.1. Local time process (occupation density function). Let $X:=\left\{X_{t}, t \geqslant 0\right\}$ be a real-valued measurable process defined on some probability space $(\Omega, \mathscr{F}, \mathbf{P})$. For any $T>0$ the occupation measure of $X$ over the interval $[0, T]$ is defined by the following path-by-path integral:

$$
\nu_{T}(B, \omega):=\int_{0}^{T} \mathbf{I}_{\left\{X_{t}(\omega) \in B\right\}} \mathrm{d} t,
$$

for any Borel set $B \subset \mathbf{R}$, where the integral is defined $\mathbf{P}$-almost everywhere with respect to $\omega \in \Omega$ (a.e.). In this paper, we say that the process $X$ admits a local time (or occupation density) on $[0, T]$ when there exists a nonnegative measurable function $(\omega, x) \mapsto l_{T}(x, \omega)$ such that

$$
\nu_{T}(B, \omega):=\int_{B} l_{T}(x, \omega) \mathrm{d} x,
$$

for $\mathbf{P}$-almost every $\omega$. The measurable process $l_{T}:=\left\{l_{T}(x), x \in \mathbf{R}\right\}$ is called the local time process of $X$ on $[0, T]$. By linearity and monotone convergence, 
the occupation time formula

$$
\int_{0}^{T} \varphi\left(X_{t}\right) \mathrm{d} t=\int_{\mathbf{R}} \varphi(x) l_{T}(x) \mathrm{d} x \quad \text { a.e. }
$$

is satisfied for any nonnegative or bounded Borel function $\varphi: \mathbf{R} \rightarrow \mathbf{R}$. For more details see, for instance, [2]-[4], [10]. In order to simplify the notation here and in what follows, we omit the term $\omega$ as usual when there is no possibility of confusion.

For a real-valued measurable stationary process, the existence of a local time implies that the marginal invariant distribution is absolutely continuous with respect to Lebesgue measure on $\mathbf{R}$.

In the semimartingale theory, the local time is usually introduced in a different way. More precisely, for a continuous semimartingale $X:=\left\{X_{t}\right.$, $t \geqslant 0\}$, the «local time» $\Lambda_{T}, T>0$, is defined by the Tanaka-Meyer formula (see [5], [7])

$$
\Lambda_{T}(x):=\left|X_{T}-x\right|-\left|X_{0}-x\right|-\int_{0}^{T} \operatorname{sgn}\left(X_{t}-x\right) \mathrm{d} X_{t},
$$

and we have

$$
\int_{0}^{T} \varphi\left(X_{t}\right) \mathrm{d}[X]_{t}=\int_{\mathbf{R}} \varphi(x) \Lambda_{T}(x) \mathrm{d} x \quad \text { a.e. }
$$

for any nonnegative or bounded measurable function $\varphi: \mathbf{R} \rightarrow \mathbf{R}$, where $[X]=\left\{[X]_{t}, t \geqslant 0\right\}$ denotes the quadratic variation process of the semimartingale $X$. In that case $\Lambda_{T}:=\left\{\Lambda_{T}(x), x \in \mathbf{R}\right\}$ is the (occupation) density of the random measure $\mathrm{d}[X]_{T}$ with respect to Lebesgue measure on $\mathbf{R}$, i.e., it is the local time process of the quadratic variation process $[X]$. If the process $X$ is a continuous semimartingale with a quadratic variation which is equal to $\mathrm{d}[X]_{t}=\sigma^{2}\left(X_{t}\right) \mathrm{d} t$ for some positive Borel function $x \mapsto \sigma^{2}(x)$, then the local time $l_{T}$ of $X$ exists and verifies

$$
l_{T}(x)=\frac{\Lambda_{T}(x)}{\sigma^{2}(x)} \quad \text { a.e. }
$$

for any $x \in \mathbf{R}$ and any $T>0$. For instance, when $X$ is a diffusion process, strong solution of the stochastic differential equation

$$
\mathrm{d} X_{t}=S\left(X_{t}\right) \mathrm{d} t+\sigma\left(X_{t}\right) \mathrm{d} W_{t}
$$

where the functions $S: \mathbf{R} \rightarrow \mathbf{R}$ and $\sigma: \mathbf{R} \rightarrow(0, \infty)$ satisfy usual conditions (16) (see Section 5 and [5], [7]), we have $\mathrm{d}[X]_{t}=\sigma^{2}\left(X_{t}\right) \mathrm{d} t$, so the local time $l_{T}$ of $X$ exists and verifies equality (4). In particular, if $X$ is a standard Brownian motion, then $l_{T}=\Lambda_{T}$.

As another class of processes with local time, we can consider the processes with absolutely continuous sample paths (see [2], [3]). Let $\{X(t)$, 
$t \geqslant 0\}$ be a measurable real process with absolutely continuous sample paths. Then the local time $l_{T}$ exists if and only if $\mathbf{P}\left[X^{\prime}(t)=0\right]=0$ for almost every $t \in[0, T]$. In this case

$$
l_{T}(x)=\sum_{t} \frac{1}{\left|X_{t}^{\prime}\right|} \mathbf{I}_{\{t \leqslant T: X(t)=x\}}
$$

We have also the following characterization of processes with local time (see [2], [4]). Let $X=\{X(t), t \geqslant 0\}$ be a measurable real process. Then $X$ admits a square integrable local time $l_{T}\left(l_{T} \in L^{2}(\mathrm{~d} x \otimes \mathbf{P})\right)$ if and only if

$$
\liminf _{\varepsilon \downarrow 0} \frac{1}{\varepsilon} \iint_{[0, T]^{2}} \mathbf{P}\left[\left|X_{s}-X_{t}\right| \leqslant \varepsilon\right] \mathrm{d} s \mathrm{~d} t<\infty .
$$

2.2. Karhunen-Loève series expansion. Let $\Phi:=\{\Phi(x), x \in \mathbf{R}\}$ be a second order, measurable, continuous in quadratic mean process on some probability space $(\Omega, \mathscr{F}, \mathbf{P})$. Then for any $M>0$ the restricted process $\{\Phi(x),|x| \leqslant M\}$ admits a Karhunen-Loève series expansion on $[-M, M]$ (see, e.g., [6, Theorem IV.3.5]). That is, for $M>0$ fixed, there exists a sequence of uncorrelated random variables $\left(\xi_{k}\right)_{k \geqslant 1}$ such that $\mathbf{E}\left[\xi_{k}^{2}\right]=\lambda_{k}$, where the $\lambda_{k} \mathrm{~s}$ are the eigenvalues associated to the eigenfunctions $\phi_{k}(\cdot)$ of the covariance operator of the restricted process $\{\Phi(x),|x| \leqslant M\}$, and such that

$$
\Phi(x)=\sum_{k=1}^{\infty} \xi_{k} \phi_{k}(x) .
$$

The series converges in quadratic mean uniformly with respect to $x \in$ $[-M, M]$. Note that the sequences $\left(\xi_{k}\right)_{k \geqslant 1},\left(\lambda_{k}\right)_{k \geqslant 1}$, and $\left(\phi_{k}(\cdot)\right)_{k \geqslant 1}$ depend on $M$. Moreover from the quadratic continuity of the process $\Phi$, the functions $\phi_{k}$ are continuous on $[-M, M]$.

Uniform convergence in probability. Assume in addition that the process $\{\Phi(x), x \in \mathbf{R}\}$ is separable and there exist $\delta_{M}>0, r=r_{M}>0$, and $c=c_{M}>0$ such that

$$
\mathbf{E}\left[|\Phi(x)-\Phi(y)|^{2}\right] \leqslant c|x-y||\ln | x-y||^{-3-r},
$$

for $|x-y| \leqslant \delta_{M},|x|,|y| \leqslant M$. Then thanks to [6, Theorem IV.3.6], series (5) converges in probability uniformly on the compact set $[-M, M]=\{x:|x| \leqslant$ $M\}$, that is, for any $\varepsilon>0$ we have

$$
\lim _{n \rightarrow \infty} \mathbf{P}\left[\sup _{|x| \leqslant M}\left|\Phi(x)-\sum_{k=1}^{n} \xi_{k} \phi_{k}(x)\right|>\varepsilon\right]=0 .
$$

Condition (6) is satisfied when there exists some $\alpha>0$ such that

$$
\mathbf{E}\left[|\Phi(x)-\Phi(y)|^{2}\right] \leqslant c|x-y|^{1+\alpha} \quad \text { if } \quad|x|,|y| \leqslant M \quad \text { and } \quad|x-y| \leqslant \delta_{M} .
$$


On the other hand, condition (6) can be weakened to

$$
\mathbf{E}\left[|\Phi(x)-\Phi(y)|^{2}\right] \leqslant c|x-y||\ln | x-y||^{-3}(\ln |\ln | x-y||)^{-1-r} .
$$

Neither condition (6) nor its weakened version (8) are necessary. Indeed, for any zero-mean measurable Gaussian process $\{\Phi(x), x \in \mathbf{R}\}$ such that

(i) $\mathbf{P}$-almost its paths are continuous, and

(ii) $(x, y) \mapsto \mathbf{E}[\Phi(x) \Phi(y)]$ is continuous,

series (5) is uniformly almost everywhere convergent on $[-M, M]$, and so satisfies relation (7) (see [12, Proposition 1.2.1, Lemma 1.2.2], see also [13]).

3. Occupation time formula. Now we establish the main result of this work. In this section $T>0$ is fixed.

Theorem 1. Let $X:=\left\{X_{t}, t \in[0, T]\right\}$ be a real-valued measurable process with a local time $l_{T}$, and let $\Phi:=\{\Phi(x), x \in \mathbf{R}\}$ be a real-valued separable measurable process which is continuous in quadratic mean, and such that

(i) $\mathbf{P}$-almost every path of the process $\Phi$ is locally bounded,

(ii) The Karhunen-Loève series expansion (5) converges uniformly on $[-M, M]$ in probability, for any $M>0$, that is, relation (7) is satisfied for any $M>0$.

Then for any bounded Borel function $g: \mathbf{R} \rightarrow \mathbf{R}$ with bounded support, the following integrals are well defined $\mathbf{P}$-almost everywhere, and satisfy the occupation time formula:

$$
\int_{0}^{T} \Phi\left(X_{t}\right) g\left(X_{t}\right) \mathrm{d} t=\int_{\mathbf{R}} \Phi(x) g(x) l_{T}(x) \mathrm{d} x \quad \text { a.e. }
$$

In particular, for any bounded Borel set $B \subset \mathbf{R}$ we have

$$
\int_{0}^{T} \Phi\left(X_{t}\right) \mathbf{I}_{\left\{X_{t} \in B\right\}} \mathrm{d} t=\int_{B} \Phi(x) l_{T}(x) \mathrm{d} x \quad \text { a.e. }
$$

Notice that there is no condition of dependency between the processes $X$ and $\Phi$.

P r o o f. The existence of the integrals in formula (9) is a direct consequence of the measurability conditions on the process $X$ and $\Phi$, and of the boundedness conditions on the paths of the process $\Phi$ and on the function $g$.

As announced to prove the equality we use the Karhunen-Loève series expansion of the process $\Phi$ combined with the classical occupation time formula (3). Assume that the support of the bounded Borel function $g: \mathbf{R} \rightarrow$ $\mathbf{R}$ is contained in $[-M, M]$ for some $M>0$, and let $n \geqslant 1$ be fixed. In Karhunen-Loève series expansion (5) the functions $\phi_{k}$ are continuous on 
$[-M, M]$, so they are bounded and we obtain that

$$
\begin{aligned}
& \left|\int_{0}^{T} \Phi\left(X_{t}\right) g\left(X_{t}\right) \mathrm{d} t-\sum_{k=1}^{n} \xi_{k} \int_{0}^{T} \phi_{k}\left(X_{t}\right) g\left(X_{t}\right) \mathrm{d} t\right| \\
& \quad \leqslant T \sup _{x}|g(x)| \cdot \sup _{|x| \leqslant M}\left|\Phi(x)-\sum_{k=1}^{n} \xi_{k} \phi_{k}(x)\right| \text { a.e. }
\end{aligned}
$$

On the other side of (9) we have

$$
\begin{aligned}
& \left|\int_{\mathbf{R}} \Phi(x) g(x) l_{T}(x) \mathrm{d} x-\sum_{k=1}^{n} \xi_{k} \int_{\mathbf{R}} \phi_{k}(x) g(x) l_{T}(x) \mathrm{d} x\right| \\
& \quad \leqslant \sup _{x}|g(x)| \cdot \sup _{|x| \leqslant M}\left|\Phi(x)-\sum_{k=1}^{n} \xi_{k} \phi_{k}(x)\right| \cdot \int_{\mathbf{R}} l_{T}(x) \mathrm{d} x \\
& \quad=T \sup _{x}|g(x)| \cdot \sup _{|x| \leqslant M}\left|\Phi(x)-\sum_{k=1}^{n} \xi_{k} \phi_{k}(x)\right| \quad \text { a.e. }
\end{aligned}
$$

The last equality is due to the fact that the integral of $x \mapsto l_{T}(x, \omega)$ on $\mathbf{R}$ is equal to $T$ for $\mathbf{P}$-almost every $\omega$.

Since the Borel function $x \mapsto \phi_{k}(x) g(x)$ is bounded, occupation time formula (3) implies for any $k$ that

$$
\int_{0}^{T} \phi_{k}\left(X_{t}\right) g\left(X_{k}\right) \mathrm{d} t=\int_{\mathbf{R}} \phi_{k}(x) g(x) l_{T}(x) \mathrm{d} x \quad \text { a.e. }
$$

Hence for any integer $n$, we have

$$
\begin{aligned}
\int_{0}^{T} & \Phi\left(X_{t}\right) g\left(X_{t}\right) \mathrm{d} t-\int_{\mathbf{R}} \Phi(x) g(x) l_{T}(x) \mathrm{d} x \\
& =\int_{0}^{T} \Phi\left(X_{t}\right) g\left(X_{t}\right) \mathrm{d} t-\sum_{k=1}^{n} \xi_{k} \int_{0}^{T} \phi_{k}\left(X_{t}\right) g\left(X_{t}\right) \mathrm{d} t \\
& +\sum_{k=1}^{n} \xi_{k} \int_{\mathbf{R}} \phi_{k}(x) g(x) l_{T}(x) \mathrm{d} x-\int_{\mathbf{R}} \Phi(x) g(x) l_{T}(x) \mathrm{d} x \quad \text { a.e. }
\end{aligned}
$$

and thanks to relations (7) and (10), we obtain that

$$
\mathbf{P}\left[\left|\int_{0}^{T} \Phi\left(X_{t}\right) g\left(X_{t}\right) \mathrm{d} t-\int_{\mathbf{R}} \Phi(x) g(x) l_{T}(x) \mathrm{d} x\right|>\varepsilon\right]=0
$$

for any $\varepsilon>0$, thus we deduce that

$$
\int_{0}^{T} \Phi\left(X_{t}\right) g\left(X_{t}\right) \mathrm{d} t=\int_{\mathbf{R}} \Phi(x) g(x) l_{T}(x) \mathrm{d} x \quad \text { a.e. }
$$

So the theorem is proved.

The hypotheses on the function $g$ can be weakened. For instance, in the following corollary we no longer assume that the support of $g$ is bounded. 
Corollary 1. Assume that the processes $\left\{X_{t}, t \in[0, T]\right\}$ and $\{\Phi(x)$, $x \in \mathbf{R}\}$ satisfy the hypotheses of Theorem 1. Then for any Borel function $g: \mathbf{R} \rightarrow \mathbf{R}$ such that $\mathbf{P}$-almost every path of the process $\left\{\Phi(x) g(x) l_{T}(x)\right.$, $x \in \mathbf{R}\}$ is integrable on $\mathbf{R}$, the occupation time formula (9) is valid.

The condition that $\mathbf{P}$-almost every path of the process $\left\{\Phi(x) g(x) l_{T}(x)\right.$, $x \in \mathbf{R}\}$ is integrable on $\mathbf{R}$ is satisfied when $g \in L^{1}(\mathbf{R})$ and $\mathbf{P}$-almost all paths of the processes $\left\{l_{T}(x), x \in \mathbf{R}\right\}$ and $\{\Phi(x), x \in \mathbf{R}\}$ are bounded on the support of the function $g$.

$\mathrm{P}$ r o o f. 1) First assume that the function $g$ is bounded and satisfies the hypotheses of the corollary. Let $g_{M}(x):=g(x) \mathbf{I}_{\{|x| \leqslant M\}}$. Thanks to Theorem 1, we have

$$
\int_{0}^{T}\left|\Phi\left(X_{t}\right) g_{M}\left(X_{t}\right)\right| \mathrm{d} t=\int_{\mathbf{R}}\left|\Phi(x) g_{M}(x)\right| l_{T}(x) \mathrm{d} x \quad \text { a.e. }
$$

Then the monotone convergence theorem applies as $M \rightarrow \infty$, and we obtain

$$
\int_{0}^{T}\left|\Phi\left(X_{t}\right) g\left(X_{t}\right)\right| \mathrm{d} t=\int_{\mathbf{R}}|\Phi(x) g(x)| l_{T}(x) \mathrm{d} x \quad \text { a.e. }
$$

Hence under the hypotheses of Corollary 1, $\mathbf{P}$-almost every path of the process $\left\{\Phi\left(X_{t}\right) g\left(X_{t}\right), t \geqslant 0\right\}$ is integrable on $[0, T]$. On the other hand, Theorem 1 allows us to write

$$
\int_{0}^{T} \Phi\left(X_{t}\right) g_{M}\left(X_{t}\right) \mathrm{d} t=\int_{\mathbf{R}} \Phi(x) g_{M}(x) l_{T}(x) \mathrm{d} x \quad \text { a.e. }
$$

and thanks to the Lebesgue dominated convergence theorem we deduce occupation time formula (9).

2) Now we consider a general function $g$ that satisfies the hypotheses of the corollary. Let $g_{n}(x):=g(x) \mathbf{I}_{\{|g(x)| \leqslant n\}}$. Then $g_{n}$ is a bounded function for which occupation time formula (9) is valid and

$$
\int_{0}^{T}\left|\Phi\left(X_{t}\right) g_{n}\left(X_{t}\right)\right| \mathrm{d} t=\int_{\mathbf{R}}\left|\Phi(x) g_{n}(x)\right| l_{T}(x) \mathrm{d} x \quad \text { a.e. }
$$

From the monotone convergence theorem, we obtain that

$$
\int_{0}^{T}\left|\Phi\left(X_{t}\right) g\left(X_{t}\right)\right| \mathrm{d} t=\int_{\mathbf{R}}|\Phi(x) g(x)| l_{T}(x) \mathrm{d} x \quad \text { a.e. }
$$

Then we can easily conclude the proof of the corollary.

$\mathrm{R}$ e $\mathrm{m}$ a r k s. 1) As we can see in the proof of Corollary 1, the condition that «P-almost every path of $\left\{\Phi(x) g(x) l_{T}(x), x \in \mathbf{R}\right\}$ is integrable on $\mathbf{R}$ » is equivalent to the condition « $\mathbf{P}$-almost every path of $\left\{\Phi\left(X_{t}\right) g\left(X_{t}\right), t>0\right\}$ is integrable on $[0, T] \gg$. 
2) Under the hypotheses of Theorem 1 assume $\mathbf{P}$-almost every path of $\{\Phi(x), x \in \mathbf{R}\}$ is bounded. Then for $g \equiv 1$, Corollary 1 applies: $\mathbf{P}$-almost every path of $\left\{\Phi\left(X_{t}\right), t \in[0, T]\right\}$ is integrable in $[0, T]$, and

$$
\int_{0}^{T} \Phi\left(X_{t}\right) \mathrm{d} t=\int_{\mathbf{R}} \Phi(x) l_{T}(x) \mathrm{d} x \quad \text { a.e. }
$$

We can also establish the following corollary.

Corollary 2. Assume that the processes $\left\{X_{t}, t \in[0, T]\right\}$ and $\{\Phi(x)$, $x \in \mathbf{R}\}$ satisfy the hypotheses of Theorem 1 . Moreover, assume that there is a positive random variable $X_{T}^{*}$ such that

$$
\sup \left\{\left|X_{t}\right|, t \in[0, T]\right\} \leqslant X_{T}^{*}<\infty \quad \text { a.e. }
$$

Then $\mathbf{P}$-almost every path of the process $\left\{\Phi\left(X_{t}\right), t \in[0, T]\right\}$ is integrable on $[0, T]$, and occupation time formula (11) is valid.

If the process $\left\{X_{t}, t \in[0, T]\right\}$ is separable and $\mathbf{P}$-almost every of its paths is bounded, then we can take $X_{T}^{*}=\sup \left\{\left|X_{t}\right|, t \in[0, T]\right\}$ which is a random variable (see, for instance, [6, Section III.2]). This is the case for the separable versions of right-continuous with left-limits processes.

P r o o f. From condition (12), there exists an increasing sequence of measurable sets $\left\{F_{n}, n \in \mathbf{N}\right\} \subset \mathscr{F}$ such that $F_{n} \subset\left\{\omega \in \Omega:\left|X_{t}(\omega)\right| \leqslant n\right.$, for all $t \in[0, T]\}$ and $\lim _{n} \mathbf{P}\left[F_{n}\right]=1$. Now consider the Borel sets $B_{n}=\{x \in$ $\mathbf{R}:|x|>n\}, n \geqslant \mathbf{N}$. Thanks to occupation time formula (3) there is $\Omega_{1}$ such that $\mathbf{P}\left[\Omega_{1}\right]=1$ and

$$
\int_{B_{n}} l_{T}(x, \omega) \mathrm{d} x=\frac{1}{T} \int_{0}^{T} \mathbf{I}_{\left\{X_{t}(\omega) \in B_{n}\right\}} \mathrm{d} t
$$

for any $\omega \in \Omega_{1}$ and any $n \in \mathbf{N}$. Furthermore if $\omega \in F_{n} \cap \Omega_{1}$, then we have

$$
\int_{B_{n}} l_{T}(x, \omega) \mathrm{d} x=\frac{1}{T} \int_{0}^{T} \mathbf{I}_{\left\{X_{t}(\omega) \in B_{n}\right\}} \mathrm{d} t=0 ;
$$

thus since the local time function $(x, \omega) \mapsto l_{T}(x, \omega)$ is nonnegative and measurable, there is a Borel set $B_{n}(\omega) \subset B_{n}$ such that $l_{T}(x, \omega)=0$ for any $x \in B_{n}(\omega)$, and the Lebesgue measure of $\bar{B}_{n}(\omega) \cap B_{n}$ is null, wher $\bar{B}_{n}(\omega)$ denotes the complementary set of $B_{n}(\omega)$.

On the one hand, from the hypotheses of the corollary, $\mathbf{P}$-almost every path of the process $\Phi$ is locally bounded, that is, there is a measurable set $\Omega_{2} \in \mathscr{F}$ such that $\mathbf{P}\left[\Omega_{2}\right]=1$ and the function $x \mapsto \Phi(x, \omega)$ is locally bounded for any $\omega \in \Omega_{2}$. Hence for each $\omega \in F_{n} \cap \Omega_{1} \cap \Omega_{2}$, we readily obtain that the function $x \mapsto \Phi(x, \omega) l_{T}(x, \omega)$ is integrable on $\mathbf{R}$ and

$$
\int_{\mathbf{R}} \Phi(x, \omega) l_{T}(x, \omega) \mathrm{d} x=\int_{\mathbf{R}} \Phi(x, \omega) l_{T}(x, \omega) \mathbf{I}_{\{|x| \leqslant n\}} \mathrm{d} x .
$$


On the other hand, thanks to Theorem 1 there is a measurable set $\Omega_{3} \in \mathscr{F}$ such that $\mathbf{P}\left[\Omega_{3}\right]=1$ and

$$
\int_{\mathbf{R}} \Phi(x, \omega) l_{T}(x, \omega) \mathbf{I}_{\{|x| \leqslant n\}} \mathrm{d} x=\int_{0}^{T} \Phi\left(X_{t}(\omega), \omega\right) \mathbf{I}_{\left\{\left|X_{t}(\omega)\right| \leqslant n\right\}} \mathrm{d} t
$$

for any $\omega \in \Omega_{3}$ and any $n \in \mathbf{N}$. Then the sequence of measurable subsets $\left\{F_{n} \cap \Omega_{1} \cap \Omega_{2} \cap \Omega_{3}, n \in \mathbf{N}\right\} \subset \mathscr{F}$ is nondecreasing and converges to some measurable set $\Omega_{0}$ such that $\mathbf{P}\left[\Omega_{0}\right]=1$. Furthermore each $\omega \in \Omega_{0}$ belongs to $F_{n} \cap \Omega_{1} \cap \Omega_{2} \cap \Omega_{3}$ for some $n \in \mathbf{N}$, and thanks to relations (13) and (14), we have

$$
\int_{0}^{T} \Phi\left(X_{t}(\omega), \omega\right) \mathrm{d} t=\int_{0}^{T} \Phi\left(X_{t}(\omega), \omega\right) \mathbf{I}_{\left\{\left|X_{t}(\omega)\right| \leqslant n\right\}} \mathrm{d} t=\int_{\mathbf{R}} \Phi(x, \omega) l_{T}(x, \omega) \mathrm{d} x .
$$

Hence occupation time formula (11) is verified. Corollary 2 is proved.

4. Convergence in distribution of ergodic means. In this section we study the convergence in distribution of weighted average (1).

Let $F_{\mathrm{b}}$ be some complete separable space of bounded real-valued Borel functions on $\mathbf{R}$ endowed with the uniform metric and closed under multiplication, that is, $f \times g \in F_{\mathrm{b}}$ for all $f, g \in F_{\mathrm{b}}$. For example, it can be $F_{\mathrm{b}}=\mathscr{C}_{\mathrm{b}}(\mathbf{R})$, the space of real-valued bounded continuous functions on $\mathbf{R}$, or $F_{\mathrm{b}}=\mathscr{C}_{0}(\mathbf{R})$, the space of real-valued continuous functions on $\mathbf{R}$ which converge to 0 at infinity, endowed with the uniform topology.

Theorem 2. Let $\left\{X_{t}, t \geqslant 0\right\}$ be a measurable process having local times $l_{T}, T>0$, and let $\Phi_{T}=\left\{\Phi_{T}(x), x \in \mathbf{R}\right\}, T>0$, be a family of measurable separable processes on $\mathbf{R}$ such that

(i) for any $T>0, \mathbf{P}$-almost every path of the process $l_{T}$ belongs to $F_{\mathrm{b}}$,

(ii) there exists some nonrandom function $f \in F_{\mathrm{b}}$ such that

$$
\lim _{T \rightarrow \infty} \frac{l_{T}}{T}=f \quad \text { in probability in } F_{\mathrm{b}},
$$

(iii) for any $T>0$, the process $\Phi_{T}$ satisfies (7) and $\mathbf{P}$-almost every of its paths is in $F_{\mathrm{b}}$,

(iv) the processes $\Phi_{T}$ converge in distribution in $F_{\mathrm{b}}$ to some measurable process $\Phi=\{\Phi(x), x \in \mathbf{R}\}$ as $T \rightarrow \infty$.

Then for any Borel function $g \in L^{1}(\mathbf{R})$ we have the following convergence in distribution in $\mathbf{R}$ :

$$
\lim _{T \rightarrow \infty} \frac{1}{T} \int_{0}^{T} \Phi_{T}\left(X_{t}\right) g\left(X_{t}\right) \mathrm{d} t=\int_{\mathbf{R}} \Phi(x) g(x) f(x) \mathrm{d} x .
$$

P r o o f. For each $T>0$, Corollary 1 applies to the processes $\left\{X_{t}, t \in\right.$ $[0, T]\}, \Phi_{T}=\left\{\Phi_{T}(x), x \in \mathbf{R}\right\}$, and the function $g$ :

$$
\frac{1}{T} \int_{0}^{T} \Phi_{T}\left(X_{t}\right) g\left(X_{t}\right) \mathrm{d} t=\int_{\mathbf{R}} \Phi_{T}(x) g(x) \frac{l_{T}(x)}{T} \mathrm{~d} x \quad \text { a.e. }
$$


Since $\Phi_{T}$ converges in distribution in the separable space $F_{\mathrm{b}}$ to $\Phi$, and $l_{T} / T$ converges in probability in $F_{\mathrm{b}}$ to a nonrandom function $f$ as $T \rightarrow \infty$, Theorems 4.4 and 5.1 in [1] entail that the product $\Phi_{T} \times l_{T} / T$ converges in distribution in $F_{\mathrm{b}}$ to $\Phi \times f$.

On the other hand, since $g \in L^{1}(\mathbf{R})$, the function $F_{\mathrm{b}} \ni h \mapsto$ $\int_{\mathbf{R}} h(x) g(x) \mathrm{d} x \in \mathbf{R}$ is continuous, $F_{\mathrm{b}}$ being endowed with the uniform topology. Then we deduce convergence (15). Theorem 2 is proved.

When the process $\left\{X_{t}, t \geqslant 0\right\}$ is stationary in law, condition (ii) corresponds to the convergence in probability in $F_{\mathrm{b}}$ of the local time estimator $l_{T} / T$ to the marginal density $f$ of the process.

In the special case when $\Phi_{T}=\Phi$ verifies (7) and $\mathbf{P}$-almost every of its paths is in $F_{\mathrm{b}}$, under conditions (i) and (ii) of Theorem 2 for the process $X$, we obtain that

$$
\lim _{T \rightarrow \infty} \frac{1}{T} \int_{0}^{T} \Phi\left(X_{t}\right) g\left(X_{t}\right) \mathrm{d} t=\int_{\mathbf{R}} \Phi(x) g(x) f(x) \mathrm{d} x \quad \text { in distribution in } \mathbf{R}
$$

for any function $g \in L^{1}(\mathbf{R})$.

We can weaken the hypotheses on $g$, provide we strengthen the conditions on the local time of the process $X$. For instance, we obtain the following result.

Corollary 3. Assume that $\left\{X_{t}, t \geqslant 0\right\}$ and $\Phi_{T}=\left\{\Phi_{T}(x), x \in \mathbf{R}\right\}$, $T>0$, satisfy conditions (i), (iii), and (iv) of Theorem 2 . In addition assume that condition (ii) is replaced by

(ii') there exist a function $f \in F_{\mathrm{b}}$ and a positive function $v \in L^{1}(\mathbf{R})$ such that

$$
\lim _{T \rightarrow \infty} \frac{l_{T}}{T v}=\frac{f}{v} \quad \text { in probability in } F_{\mathrm{b}} .
$$

Then for any Borel function $g: \mathbf{R} \rightarrow \mathbf{R}$ such that $g \times v \in L^{1}(\mathbf{R})$, the convergence in distribution (15) holds. In particular, the average of $\Phi_{T}\left(X_{t}\right)$ on $[0, T]$ converges in distribution as $T \rightarrow \infty$ :

$$
\lim _{T \rightarrow \infty} \frac{1}{T} \int_{0}^{T} \Phi_{T}\left(X_{t}\right) \mathrm{d} t=\int_{\mathbf{R}} \Phi(x) f(x) \mathrm{d} x \quad \text { in distribution in } \mathbf{R} .
$$

P r o o f. From the hypotheses on the processes $\Phi_{T}, T \geqslant 0$, Corollary 1 applies and we can write

$$
\begin{aligned}
\frac{1}{T} \int_{0}^{T} \Phi_{T}\left(X_{t}\right) g\left(X_{t}\right) \mathrm{d} t & =\int_{\mathbf{R}} \Phi_{T}(x) g(x) \frac{l_{T}(x)}{T} \mathrm{~d} x \\
& =\int_{\mathbf{R}} \Phi_{T}(x) g(x) \frac{l_{T}(x)}{T v(x)} v(x) \mathrm{d} x .
\end{aligned}
$$

On the other hand, the function $F_{\mathrm{b}} \ni h \mapsto \int_{\mathbf{R}} h(x) g(x) v(x) d x \in \mathbf{R}$ is continuous, hence with the convergence

$$
\lim _{T \rightarrow \infty} \frac{\Phi_{T} \times l_{T}}{T v}=\frac{\Phi \times f}{v} \text { in distribution in } F_{\mathrm{b}},
$$


we conclude the proof of the corollary.

Remark that with condition (ii'), it is assumed that $l_{T} / v, f / v \in F_{\mathrm{b}}$. If the function $v$ is bounded, then

$$
\sup _{x}\left|\frac{l_{T}(x)}{T}-f(x)\right| \leqslant \sup _{x}\left|\frac{l_{T}(x)}{T v(x)}-\frac{f(x)}{v(x)}\right| \sup _{x}|v(x)|,
$$

and condition (ii') implies condition (ii).

Next let $F$ be some complete separable metric space of locally bounded real-valued Borel functions on $\mathbf{R}$ endowed with the local uniform topology and closed by multiplication. The space $F$ can be any complete separable subspace of the space $D(\mathbf{R})$ of càdlàg real-valued functions defined on $\mathbf{R}$ endowed with the local uniform topology. For example, $F=\mathscr{C}(\mathbf{R})$, the space of real-valued continuous functions on $\mathbf{R}$. In this case Theorem 2 remains valid when we replace the functional space $F_{\mathrm{b}}$ by $F$, provided the Borel function $g \in L^{1}(\mathbf{R})$ has bounded support.

Corollary 4. Let $\left\{X_{t}, t \geqslant 0\right\}$ be a measurable process having local times $l_{T}, T>0$, and let $\Phi_{T}=\left\{\Phi_{T}(x), x \in \mathbf{R}\right\}, T>0$, be a family of measurable separable processes on $\mathbf{R}$ such that

(i) for any $T>0, \mathbf{P}$-almost all paths of the process $l_{T}$ belong to $F$,

(ii) there exists some nonrandom function $f \in F$ such that

$$
\lim _{T \rightarrow \infty} \frac{l_{T}}{T}=f \quad \text { in probability in } F,
$$

(iii) for any $T>0$, the process $\Phi_{T}$ satisfies (7) and $\mathbf{P}$-almost every of its paths is in $F$,

(iv) the processes $\Phi_{T}$ converge in distribution in $F$ to some measurable process $\Phi=\{\Phi(x), x \in \mathbf{R}\}$ as $T \rightarrow \infty$.

Then for any Borel function $g \in L^{1}(\mathbf{R})$ with bounded support, convergence (15) holds.

P r o o f. For each $T>0$, Corollary 1 applies to the processes $\left\{X_{t}, t \in\right.$ $[0, T]\}, \Phi_{T}=\left\{\Phi_{T}(x), x \in \mathbf{R}\right\}$, and the function $g$. Thus

$$
\frac{1}{T} \int_{0}^{T} \Phi_{T}\left(X_{t}\right) g\left(X_{t}\right) \mathrm{d} t=\int_{\mathbf{R}} \Phi_{T}(x) g(x) \frac{l_{T}(x)}{T} \mathrm{~d} x \quad \text { a.e. }
$$

Since $\Phi_{T}$ converges in distribution in the separable space $F$ to $\Phi$, and $l_{T} / T$ converges in probability in $F$ to a nonrandom function $f$ as $T \rightarrow \infty$, Theorem 4.4 and 5.1 in [1] entail that the product $\Phi_{T} \times l_{T} / T$ converges in distribution in $F$ to $\Phi \times f$.

On the other hand, since $g \in L^{1}(\mathbf{R})$ with bounded support, the function $F \ni h \mapsto \int_{\mathbf{R}} h(x) g(x) \mathrm{d} x \in \mathbf{R}$ is continuous, the space $F$ being endowed with the local uniform topology. The we can readily deduce convergence (15). Corollary 4 is probed. 
5. Application to the estimation of the marginal density of a diffusion process. Let $X:=\left\{X_{t}, t \in \mathbf{R}\right\}$ be a strong solution of the following stochastic differential equation:

$$
\mathrm{d} X_{t}=S\left(X_{t}\right) \mathrm{d} t+\sigma\left(X_{t}\right) \mathrm{d} W_{t},
$$

where

$$
\limsup _{|x| \rightarrow \infty} \operatorname{sgn}(x) S(x)<0 \quad \text { and } \quad 0<\sigma_{1} \leqslant \sigma(x) \leqslant \sigma_{2}<\infty .
$$

It is well known that under these conditions the diffusion process $X$ admits a marginal invariant distribution $\mu$ with density function $f$ with respect to Lebesgue measure on $\mathbf{R}$. Assume that $X_{0}$ follows this invariant distribution, that is, the diffusion process is stationary with marginal distribution $\mu$. In what follows, we also need the two conditions

$$
\mathbf{E}\left[\left(\frac{F\left(X_{0} \wedge x\right)-F\left(X_{0}\right) F(x)}{f\left(X_{0}\right)}\right)^{2}\right]<\infty
$$

and

$$
\mathbf{E}\left[\left(f(x) \frac{\mathbf{I}_{\left\{X_{0}>x\right\}}-F\left(X_{0}\right)}{f\left(X_{0}\right)}\right)^{2}\right]<\infty .
$$

In the estimation problem of the invariant distribution function $F(x)=$ $\mu(-\infty, x]=\mathbf{P}\left[X_{t} \leqslant x\right]$, I. Negri [11] has studied the empirical distribution function

$$
\widehat{F}_{T}(x):=\frac{1}{T} \int_{0}^{T} \mathbf{I}_{\left\{X_{t} \leqslant x\right\}} \mathrm{d} t
$$

which is a consistent estimator of $F(x)$. In [11, Lemma 5.2] it is shown that the processes $\zeta_{T}:=\left\{\zeta_{T}(x):=\sqrt{T}\left(\widehat{F}_{T}(x)-F(x)\right), x \in \mathbf{R}\right\}$ converge in distribution in $\mathscr{C}_{0}(\mathbf{R})$ to a zero-mean Gaussian process $\zeta$ as $T$ goes to $\infty$. By the way it was proved that for any $M>0$, there is a constant $c>0$ such that

$$
\mathbf{E}\left[\left|\zeta_{T}(x)-\zeta_{T}(y)\right|^{2}\right] \leqslant c|x-y|^{2}, \quad|x|,|y| \leqslant M .
$$

On the other hand, the ratio $l_{T} / T$ is a consistent estimator of the density function $f$, and in $\left[8\right.$, Theorem 5] it is proved that the processes $\eta_{T}:=$ $\left\{\eta_{T}(x):=\sqrt{T}\left(l_{T}(x) / T-f(x)\right), x \in \mathbf{R}\right\}$ converge in distribution in $\mathcal{C}_{0}(\mathbf{R})$ to a zero-mean Gaussian process $\eta$ as $T$ goes to $\infty$. Thus Theorem 2 applies and we deduce the following result.

Proposition 1. For any $g \in L^{1}(\mathbf{R})$ we have

$$
\begin{aligned}
\lim _{T \rightarrow \infty} & \frac{1}{\sqrt{T}} \int_{0}^{T}\left(\widehat{F}_{T}\left(X_{t}\right)-F\left(X_{t}\right)\right) g\left(X_{t}\right) \mathrm{d} t \\
& =\int_{\mathbf{R}} \zeta(x) g(x) f(x) \mathrm{d} x \quad \text { in distribution in } \mathbf{R} .
\end{aligned}
$$


In particular, for any bounded Borel set $B \subset \mathbf{R}$ we have the following convergence in distribution in $\mathbf{R}$ :

$$
\lim _{T \rightarrow \infty} \frac{1}{\sqrt{T}} \int_{0}^{T}\left(\widehat{F}_{T}\left(X_{t}\right)-F\left(X_{t}\right)\right) \mathbf{I}_{\left\{X_{t} \in B\right\}} \mathrm{d} t=\int_{B} \zeta(x) f(x) \mathrm{d} x .
$$

Coming back to the general case of this section, Proposition 1 together with the ergodicity of the diffusion process $X$ imply that

$$
\lim _{T \rightarrow \infty} \frac{1}{T} \int_{0}^{T} F\left(X_{t}\right) g\left(X_{t}\right) \mathrm{d} t=\mathbf{E}[F(\xi) g(\xi)]=\int_{\mathbf{R}} F(x) g(x) f(x) \mathrm{d} x \quad \text { a.e., }
$$

where $\xi$ is a random variable which distribution is the invariant distribution $\mu$. Following [8] and [11], we state in Proposition 2 (see Annexe) that the 2 -dimensional processes $\left(\zeta_{T}, \eta_{T}\right)$ converges in distribution in $\mathscr{C}_{0}(\mathbf{R}) \times \mathscr{C}_{0}(\mathbf{R})$ to a 2 -dimensional zero-mean Gaussian process $(\zeta, \eta)$ as $T$ goes to $\infty$. Hence whenever $g \in L^{1}(\mathbf{R})$, we deduce that

$$
\begin{aligned}
\Delta_{T}(g):= & \sqrt{T}\left(\frac{1}{T} \int_{0}^{T} \widehat{F}_{T}\left(X_{t}\right) g\left(X_{t}\right) \mathrm{d} t-\int_{\mathbf{R}} F(x) g(x) f(x) \mathrm{d} x\right) \\
= & \frac{1}{T} \int_{0}^{T} \zeta_{T}\left(X_{t}\right) g\left(X_{t}\right) \mathrm{d} t \\
& +\sqrt{T}\left(\frac{1}{T} \int_{0}^{T} F\left(X_{t}\right) g\left(X_{t}\right) \mathrm{d} t-\int_{\mathbf{R}} F(x) g(x) f(x) \mathrm{d} x\right) \\
= & \int_{\mathbf{R}}\left(\zeta_{T}(x) \frac{l_{T}(x)}{T}+F(x) \eta_{T}(x)\right) g(x) \mathrm{d} x .
\end{aligned}
$$

Since the processes $l_{T} / T$ converge in probability in $\mathscr{C}_{0}(\mathbf{R})$ to the nonrandom function $f$ as $T$ goes to $\infty$, we deduce that the processes $\left\{\zeta_{T}(x) l_{T}(x) / T+F(x) \eta_{T}(x), x \in \mathbf{R}\right\}$ converges in distribution in $\mathscr{C}_{0}(\mathbf{R})$ to $\{\zeta(x) f(x)+F(x) \eta(x), x \in \mathbf{R}\}$ as $T$ goes to $\infty$. For $g \in L^{1}(\mathbf{R})$, the function $\mathscr{C}_{0}(\mathbf{R}) \ni h \mapsto \int_{\mathbf{R}} h(x) g(x) \mathrm{d} x \in \mathbf{R}$ is continuous. These imply that the random variables $\Delta_{T}(g)$ converge in distribution to a zero-mean Gaussian random variable

$$
\int_{\mathbf{R}} \zeta(x) g(x) f(x) \mathrm{d} x+\int_{\mathbf{R}} F(x) g(x) \eta(x) \mathrm{d} x=\int_{\mathbf{R}}(\zeta(x) f(x)+F(x) \eta(x)) g(x) \mathrm{d} x
$$

as $T$ goes to infinity. Finally we obtain the following central limit theorem.

Theorem 3. For any $g \in L^{1}(\mathbf{R})$ we have

$$
\begin{aligned}
\lim _{T \rightarrow \infty} & \sqrt{T}\left(\frac{1}{T} \int_{0}^{T} \widehat{F}_{T}\left(X_{t}\right) g\left(X_{t}\right) \mathrm{d} t-\int_{\mathbf{R}} F(x) g(x) f(x) \mathrm{d} x\right) \\
= & \int_{\mathbf{R}}(\zeta(x) f(x)+F(x) \eta(x)) g(x) \mathrm{d} x
\end{aligned}
$$

in distribution in $\mathbf{R}$. 
In particular, for any bounded Borel set $B$ of $\mathbf{R}$ we have the following convergence in distribution in $\mathbf{R}$ :

$$
\begin{aligned}
\lim _{T \rightarrow \infty} & \sqrt{T}\left(\frac{1}{T} \int_{0}^{T} \widehat{F}_{T}\left(X_{t}\right) \mathbf{I}_{\left\{X_{t} \in B\right\}} \mathrm{d} t-\int_{B} F(x) f(x) \mathrm{d} x\right) \\
= & \int_{B}(\zeta(x) f(x)+F(x) \eta(x)) \mathrm{d} x .
\end{aligned}
$$

Annexe: Convergence in distribution of $\left(\zeta_{T}, \eta_{T}\right)$. To complete the proof of Theorem 3, it remains to establish the convergence of $\left(\zeta_{T}, \eta_{T}\right)$.

Proposition 2. The two-dimensional processes $\left(\zeta_{T}, \eta_{T}\right):=\left\{\left(\zeta_{T}\left(x_{1}\right)\right.\right.$, $\left.\left.\eta_{T}\left(x_{2}\right)\right), x_{1} \in \mathbf{R}, x_{2} \in \mathbf{R}\right\}$ converge in distribution in $\mathscr{C}_{0}(\mathbf{R}) \times \mathscr{C}_{0}(\mathbf{R})$ to a two-dimensional zero-mean Gaussian process as $T$ tends to $\infty$.

P r o o f. In [8] and [11], it is proved that the processes $\left\{\zeta_{T}(x), x \in \mathbf{R}\right\}$ and $\left\{\eta_{T}(x), x \in \mathbf{R}\right\}$ are convergent in distribution in $\mathscr{C}_{0}(\mathbf{R})$. Here, we establish the joint convergence. For simplicity of exposition we assume that $\sigma(x) \equiv 1$.

From [8, relation (14)] and [11] (see also relations (1.32) and (1.130) in [9]) we can write

$$
\begin{aligned}
\zeta_{T}(x):= & \sqrt{T}\left(\widehat{F}_{T}(x)-F(x)\right)=\frac{1}{\sqrt{T}} \int_{0}^{T}\left(\mathbf{I}_{\left\{X_{t} \leqslant x\right\}}-F(x)\right) \mathrm{d} t \\
= & \frac{2}{\sqrt{T}} \int_{X_{0}}^{X_{T}} \frac{F(v \wedge x)-F(v) F(x)}{f(v)} \mathrm{d} v \\
& -\frac{2}{\sqrt{T}} \int_{0}^{T} \frac{F\left(X_{t} \wedge x\right)-F\left(X_{t}\right) F(x)}{f\left(X_{t}\right)} \mathrm{d} W_{t} \\
= & Y_{1, T}(x)-Z_{1, T}(x)
\end{aligned}
$$

and

$$
\begin{aligned}
\eta_{T}(x) & :=\sqrt{T}\left(\frac{l_{T}(x)}{T}-f(x)\right) \\
& =\frac{2 f(x)}{\sqrt{T}} \int_{X_{0}}^{X_{T}} \frac{\mathbf{I}_{\{v>x\}}-F(v)}{f(v)} \mathrm{d} v-\frac{2 f(x)}{\sqrt{T}} \int_{0}^{T} \frac{\mathbf{I}_{\left\{X_{t}>x\right\}}-F\left(X_{t}\right)}{f\left(X_{t}\right)} \mathrm{d} W_{t} \\
& =Y_{2, T}(x)-Z_{2, T}(x) .
\end{aligned}
$$

So for the convergence in distribution of $\left(\zeta_{T}, \eta_{T}\right)=\left(Y_{1, T}, Y_{2, T}\right)-$ $\left(Z_{1, T}, Z_{2, T}\right)$, it suffices to establish the convergence in probability in $\mathscr{C}_{0}(\mathbf{R}) \times$ $\mathscr{C}_{0}(\mathbf{R})$ of $\left(Y_{1, T}, Y_{2, T}\right)$ to $(0,0)$, and the convergence in distribution in $\mathscr{C}_{0}(\mathbf{R}) \times \mathscr{C}_{0}(\mathbf{R})$ of $\left(Z_{1, T}, Z_{2, T}\right)$ as $T$ tends to $\infty$.

The convergence of $\left(Y_{1, T}, Y_{2, T}\right)$ to $(0,0)$ is a direct consequence of the convergence of each of the components obtained in [8] and [11].

We establish the convergence in distribution of $\left(Z_{1, T}, Z_{2, T}\right)$ in $\mathscr{C}_{0}(\mathbf{R}) \times$ $\mathscr{C}_{0}(\mathbf{R})$ by showing the convergence of the finite dimensional distributions of 
the processes, and by using the uniform tightness of the family of distributions.

For the finite dimensional convergence in distribution we will use the Cramér-Wold device. Let $n>0$ and let $x_{1}, \ldots, x_{n}, y_{1}, \ldots, y_{n}, \alpha_{1}, \ldots, \alpha_{n}$, $\beta_{1}, \ldots, \beta_{n}$ be in $\mathbf{R}$. Consider the random variable

$$
z_{T}=\sum_{i=1}^{n}\left(\alpha_{i} Z_{1, T}\left(x_{i}\right)+\beta_{i} Z_{2, T}\left(y_{i}\right)\right)=\frac{2}{\sqrt{T}} \int_{0}^{T} g\left(X_{t}\right) \mathrm{d} t
$$

where

$$
g(x)=\sum_{i=1}^{n}\left(\alpha_{i} \frac{F\left(x \wedge x_{i}\right)-F(x) F\left(x_{i}\right)}{f(x)}+\beta_{i} f\left(y_{i}\right) \frac{\mathbf{I}_{\left\{x>y_{i}\right\}}-F(x)}{f(x)}\right) .
$$

The diffusion process $\left\{X_{t}, t \geqslant 0\right\}$ is stationary and we can easily prove the following inequality:

$$
\begin{aligned}
\rho^{2}:= & \mathbf{E}\left[g\left(X_{t}\right)^{2}\right] \\
\leqslant & 2 n \sum_{i=1}^{n} \alpha_{i}^{2} \mathbf{E}\left[\left(\frac{F\left(X_{t} \wedge x_{i}\right)-F\left(X_{t}\right) F\left(x_{i}\right)}{f\left(X_{t}\right)}\right)^{2}\right] \\
& +2 n \sum_{i=1}^{n} \beta_{i}^{2} \mathbf{E}\left[\left(f\left(y_{i}\right) \frac{\mathbf{I}_{\left\{X_{t}>y_{i}\right\}}-F\left(X_{t}\right)}{f\left(X_{t}\right)}\right)^{2}\right] .
\end{aligned}
$$

By hypotheses the previous expression is finite. Since the diffusion process $\left\{X_{t}, t \geqslant 0\right\}$ is ergodic, the central limit theorem for Wiener integral (see [9, Theorem 1.19]) can be applied, and we deduce that the distribution of the random variable $z_{T}$ converges to the normal distribution $\mathscr{N}\left(0,4 \rho^{2}\right)$ as $T$ tends to $\infty$. Consequently, the finite dimensional distributions of the process $\left(Z_{1, T}, Z_{2, T}\right)$ converge.

The tightness of the family of distributions of the processes $\left(Z_{1, T}, Z_{2, T}\right)$, $T>0$, comes readily from the tightness of the two families of marginal distributions, that is, the two families of distributions of the processes $Z_{1, T}$, $T>0$, and the processes $Z_{2, T}, T>0$ (see [8] and [11]). This completes the proof of the proposition.

Acknowledgments. The author wishes to thank an anonymous referee for helpful comments and suggestions leading to an essential improvement of the manuscript.

\section{REFERENCES}

1. Биллингсли П. Сходимость вероятностных мер. М.: Наука, 1977, 351 с.

2. Bosq D., Davydov Y. Local time and density estimation in continuous time. - Math. Method. Statist., 1999, v. 8, № 1, p. 22-45. 
3. Geman D., Horowitz J. Occupation times for smooth stationary processes. - Ann. Probab., 1973, v. 1, № 1, p. 131-137.

4. Geman D., Horowitz J. Occupation densities. — Ann. Probab., 1980, v. 8, № 1, p. 167.

5. Гихман И. И., Скороход А.В. Стохастические дифференциальные уравнения. Киев: Наукова думка, 1968, 354 с.

6. Гихман И. И., Скороход А. В. Теория случайных процессов, т. 1. М.: Наука, 1971, $664 \mathrm{c}$.

7. Karatzas I., Shreve S.E. Brownian Motion and Stochastic Calculus. New York: Springer-Verlag, 1988, $470 \mathrm{p}$.

8. Kutoyants Yu.A. Efficient density estimation for ergodic diffusion processes. Statist. Inference Stoch. Process., 1998, v. 1, № 2, p. 131-155.

9. Kutoyants Yu.A. Statistical Inference for Ergodic Diffusion Processes. London: Springer-Verlag, 2004, 481 p.

10. Насыров Ф. С. О локальных временах для функций случайных процессов. I, II. Теория вероятн. и ее примен., 1995 , т. 40, в. 4, с. $798-812 ; 1996$, т. 41 , в. 2 , с. $284-$ 299.

11. Negri I. Stationary distribution function estimation for ergodic diffusion process. Statist. Inference Stoch. Process., 1998, v. 1, № 1, p. 61-84.

12. Pycke J.-R. Un lien entre le développement de Karhunen-Loève de certains processus gaussiens et le laplacien dans des espaces de Riemann. - Mémoire de thèse de doctorat de l'Université Paris 6, 2003.

13. Pycke J.-R. Explicit Karhunen-Loève expansions related to the Green function of the Laplacian. - Banach Center Publ., 2006, v. 72, p. 263-270.

Поступила в редакцию 29.VI. 2010

Исправленный вариант 